\title{
XI.
}

\section{Ueber die Wurzel der Rubia tinctorum.}

\author{
Yon \\ Prof. Rochleder.
}

(Sitzungsber. d. kais. Akad. d. Wissensch. 2. Wien v. 11. Decbr. 1852.)

\section{Tweite Abhandlung.*)}

Ich habe in der ersten Abhandlung unter dem Namen Ruberythrinsāure eine Substanz beschrieben, welche sich durch ihr Verhalten gegen Säuren und Alkalien auszeichnet, durch deren Einwirkung sie sich in Zucker und Alizarin spaltet. Die Substanz war zur Analyse im luftleeren Raum über Schwefelsăure getrocknet. Ich habe aus Gründen, die ich weiter unten auseinandersetzen werde, vermuthet, dass die bei gewő̈hnlicher Temperatur im luftleeren Raum getrocknete Substanz noch Wasser enthalte und eine neu largestellte Menge derselben bei $100^{\circ} \mathrm{C}$. getrocknet.

Herr R. Schwarz erhielt bei der Analyse derselben von 0,2365 Substanz 0,4720 Kohlensäure, d. i. 54,42 p. C. Kohlenstoff. Die im Vacuo getrocknete Substanz gab 54,54 p. C. Kohle. Es lässt sich also bei $100^{\circ} \mathrm{C}$. aus der Rubergthrinsăure kein Wasser weiter austreiben.

Ich habe mich vergebens bemüht, die Spaltung der Ruberythrinsãure in Alizarin und Zucker durch Emulsin zu bewerkstelligen, die Säure blieb unverändert. Dieses negative Resultat, bei Anwendung des Fermentes der Mandein ist natürlich kein Beweis, dass nicht im krapp oder andern Ptlanzen Fermente enthalten sein können, welche diese Spaltung mit Leichtigkeit bewerkstelligen.

Ich habe es für ganz überflüssig gefunden, Versuche über die Zusammensetzung des Alizarin und Purpurin anzustellen, da diese beiden Stoffe durch die Arbeiten von Schunk, Debus, Strecker und Wolff ohnedies genauer gekannt sind, als es mit den übrigen Bestandtheilen der Wurzel der Fall war.

*) VgL. d. Journ. LV, 385. 
Ich halte es nicht für überflüssig, auf den in die Augen fallenden Zusammenhang hinzuweisen, der zwischen dem Alizarin und einem Stoffe zu bestehen scheint, den ich vor neun Jahren gemeinschanlich mit Herrn Held aus Parmelia parietina dargestellt und Chrysophansiure genannt habe. Nach den jetzt geltenden Atomgewichten ${ }^{*}$ ) berechnet, ist die Zusammensetzung dieser Säure nach den damals angestellten Analyssen **) folgende:

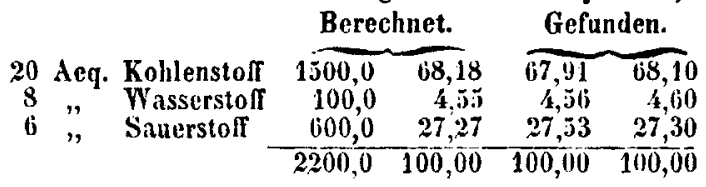

Dieser Farbstoff wurde später von Schlossberger und Dupping in der Wuzel der Rhabarber gefunden und mit denselben Resultaten analysirt.

Die Formel $\mathrm{C}_{20} \mathrm{II}_{5} \mathrm{O}_{6}$ lïsst sich, wie schon damals bemerkt wurde, betrachten als Naphtalin $\mathrm{C}_{20} \mathrm{II}_{8}$ melsr 6 Aeq. Sauerstoff. Als Hydrat betrachtet, ist seine Formel $\mathrm{C}_{20} \mathrm{Il}_{7} \mathrm{O}_{5}+\mathrm{H} 0$. Dieser Formel nach unterschcidet sich die Chrysophansäture von dem Alizarin durch ein Aerguivalent Wasserstoff, das sie mehr, und ein Aequivalent Saucrstoff, das sie weniger enthält als letzteres. $\mathrm{C}_{20} \mathrm{H}_{5} \mathrm{O}_{5}=\mathrm{C}_{20} \mathrm{II}_{6} \mathrm{O}_{6}+\mathrm{II}-0$. Demnach bestünde derselbe $\mathrm{Zu}-$ sammenhang zwischen Clorysophansäure und Alizarin wie zwischen Aldehyd und Essigsiure.

Der nahe Zusammenhang, der durch die Zusammensetzung angedeutet wird, erhält noch weiter in hohem Grade Wahrscheinlichkeit durch die grosse Aehnlichkeit in den Eigenschaften dieser beiden Körper. Ich setze zur leichteren Uebersicht die entsprechenden Eigenschaften beiler Stoffe neben einander.

Chrysophansäure.

Goldgelbe Nadeln.

Bei hơherer Temperatur unter Zurücklassung von Kohle theilweise unzersetzt llüchtig.

\section{Aliæarin.}

Orangegelbe Nadeln.

Bei höherer Temperatur unter Zurücklassung von Kohle theilweise unzersetzt flüchtig.

") $\mathrm{C}=75 ; \mathrm{H}=12,5$.

**) Annalen der Chemie und Pharmacie von F. Wo hler und J. Liebig, Band XLVII, p. 13. 
Chrysophansäure.

Beinahe unlöslich in kaltem, wenig löslich in siedendem Wasser.

Nit intensiv - gelber Farbe in Alkohol und Aether löslich.

Mit blutrother Farbe in concentritter Schwefelsüure löslich, whue Zersetzung. Durch Zusatz von Wasser aus der schivefelsauren Lōsung in gelben Flocken unverindert fillbar.

Löslich in Kali und Natronlüsung mit prachtvol! rolher Farbe. Die Losungen werden durch Situren unter $\Lambda$ bscheidung gelber Flocken enträrbt.

Die Auflüsung in Ammonials ist blutroth.

Die trockene Verbindung mit Kali ist indigblau.
Alizarin.

Unlüslich in kaltem, wenig löslich in heissem Wasser.

Mit dunkelgelber Farbe in Alkohol und Aether löslich.

Lỏslich mit rother Farbe in concentrirter Schwefelsäure. Die Lōsung lāsst auf Zusatz von Wasser das Alizarin unverandert in gelben Flocken fallen.

Lüslich in Kalilauge mit prachtroll rother Farbe in durchfallendem, reilchenblaner Farbe in reflectirtem Lichte. Die Lüsung vird durch Säuren unter Füllung gelber Flocken enträrbt.

Die ammoniakalische Lösung ist purpurroth.

Die Kali- und Baryt-Verbindung ist dunkelviolett.

Das Bleisalz der Chrysophansïture ist zinnoberroth wie das Bleisalz der Rnberythrinsäure. Die alkalischen Lösungen der Chrysophansiure sind von denen des Purpurin nicht zu unterscheiden.

Die Zahlen, wclche Debus bei der Analyse des Purpurin und der Bleiverbindung desselben erhielt, führten ihn zur Formel $\mathrm{C}_{15} \mathrm{H}_{5} \mathrm{O}_{5}$ und $\mathrm{C}_{15} \mathrm{II}_{4} \mathrm{O}_{4}, \mathrm{PbO}$. Strecker und $\mathrm{W}_{0}$ olf berechnen dalür dic Formeln $\mathrm{C}_{18} \mathrm{H}_{6} \mathrm{O}_{6}$ und $5\left(\mathrm{C}_{13} \mathrm{II}_{5} \mathrm{O}_{5}\right)+6 \mathrm{~Pb} 0=$ $4\left(\mathrm{C}_{18} \mathrm{HI}_{3} \mathrm{O}_{5}, \mathrm{PbO}\right)+\mathrm{C}_{18} \mathrm{II}_{5} \mathrm{O}_{3}, 2 \mathrm{PbO}$.

Das Purpurin giebt bei der Oxydation nach Strecker und Wo!ff dieselben Producto wie das Alizarin, Oxalsäure und Phtalsäure. Diese beiden Chemiker führen an, dass ein Unterschied von 2 Aeqr. Kohlenstoff bei zwei nahe verwandten Körpern ein ungewöhnliches Verhältniss sei. 
Diese Anomalie fallt weg, wenn man für das Purpurin die Formel $\mathrm{C}_{60} \mathrm{H}_{20} \mathrm{O}_{20}$ annimmt. Die Formel der Bleiverbinduug von $\mathrm{Deb}$ u s ist dann $=\mathrm{C}_{60} \mathrm{H}_{16} \mathrm{O}_{16}+4 \mathrm{PbO}$. Es sind vier Aequivalente Wasser durch vier Aequivalente Bleioxyd ersetzt.

Das Purpurin würıle nach dieser Formel sich von dem Alizarin durch ein drei Mal so hohes Atomgewicht und 2 Aeq. Wasser unterscheiden, die es im freien Zustande mehr enthielte, als das Alizarin. Daraus wäre es erklärlich, warum es bei der Oxydation dieselben Producte wie das Alizarin liefert.

Ein Aequivalent Purpurin würde in drei Aeq. Alizarin unter Verlust von zwei Aequivalenten Wasser zerfallen, dieses Alizarin würde durch Zufuhr von Sauerstoff in Oxalsăure und Phtalsãure zerfallen.

Strecker und Wolff hahen aus gegohrenem Krapp Purpurin frei von Alizarin erhalten. Es wäre denkbar, dass die Bildung von Purpurin liebei analog der Bildung des Kartoffelfuselöles bei der Weingährung vor sich grgangen wäre. Wie Liebig in seinen ,chemischen Briefen" bemerkt, künnen 2 Aeণq. Kartoffelfuselöl gehildet werden, indem 5 Aeq. Alkohol, 6 Aeq. Wasserstoff und Sauerstoff in der Form von Wasser abgeben. Wenn die Ruberythrinsăure bei der Gährung des Krappes sich zerlegt, können statt 1 Aeq. Zucker und 3. Aeq. Alizarin, Purpurin und Alkohol und Kohlensäure oder Purpurin und Milchsăure entstehen. Drei Aequivalente Alizarin $=\mathrm{C}_{60} \mathrm{H}_{18} \mathrm{O}_{18}$ künnen unter Abgabe ron 2 Aer. Wasserstoff und Sauerstoff ein Aeq. wassertreies Purpurin erzeugen.

Ich habe mich überzeugt, dass Krapp, an feuchten Orten aufbewalurt, wo er oberflächlich zu schimmeln begann, viel weniger Ruberythrinsäure enthielt, als zuvor.

Ich gehe nun auf eine andere Substanz über, die mit der Ruberythrinsãure im năchsten Zusammenhang steht, wenn sie nicht damit identisch ist. Anders on bat vor einigen Jabren eine Untersuchung über einige Farbstoffe der Morinda citrifolia (aus der Familie der Rubiaceae) veroffentlicht. *) Er fand in der Wurzel dieser Pflanze, die in Ostindien zum Färben dient, einen gelben Farbstofl, den er Morindin nannte, der durch Schwefel-

*) S. d. Journ. XIVII, 431. 
säure und trockne Destillation in einen andern Farbstoff, das Morindon, übergeführt werden konnte. Dieses Morindon scheint nichts als Alizarin zu sein, das Morindin dagegen Ruberythrinsãure. Anders on stellte für das Morindin die Formel $\mathrm{C}_{28} \mathrm{H}_{15} \mathrm{O}_{15}$, für das Morindon die Formel $\mathrm{C}_{28} \mathrm{H}_{10} \mathrm{O}_{10}$ auf. Demnach entstünde das Morindon aus Morindin durch Verlust von 5 Aeq. Wasser. Ich stelle hier wieder die Eigenschaften des Morindin, wie sie Anderson beobachtete, neben die entsprechenden Eigenschaften der Ruberythrinsăure, die ich in dem ersten Theile dieser Ablandlung beschrieben habe.

\section{Morindin.}

Das Morindin krystallisirt aus Alkohol in feinen Nadeln von gelber Farbe.

Wird von Alkalilüsungen mit rother Farbe aufgenommen.

Löslich in concentrirter Schwefelsãure, durch Wasser werden gelbe Flocken gefältt, die sich in Alkalien mit violetter Farbe lösen.

\section{Ruberythrinsäure.}

Ebenso.

Ebenso.

Ebenso.

Ebenso.

Mit basisch-essigsaurem Bleioxyd flockiger, scharlachrother Niederschlag.

Kalk- und Barytwasser geben voluminüse rothe Niederschlāge.

Eisenchloridlüsung giebt keinen Niederschlag, die Lösung ist braun.

Die ammoniakalische Lỏsung giebt mit Alaunlossung einen ro-
then Lack.

Beim Erbitzen entsteht gelbrother Dampf, der sich zu rothen Nadeln verdichtet, die in Alkalien mit violetter Farbe sich lōsen. Es bleibt Kohle zurūck. Ebenso.

Ebenso.

Ebenso.

Ebenso. 
Anders on hat mit dem Morindin Zeuge, die mit Alaunoder Eisenbeize versehen waren, nicht färben können, eben so wenig ist ein Fürben solcher gebeizter Zeuge mit Ruberythrinsäure möglich. Der einzige Unterschied zwischen Morindin und Rubergtbrinsäure ist der, dass Morindlin nach A nderson aus einer sietlenden, wässerigen Lüsung sich in gallertigen Flocken ausscheidet, dic keine Anzeichen von Krystallisation zeigen und beim Filtriren das Filter verstopfen, während eine heisse Lösung von Ruberythrinsüure beim Frrkalten Flocken fallen lässt, die aus Krystallen bestehen.

Das Norindin gab bei der Analyse, dic $\Lambda \mathrm{nders}$ on damit anstelle, $5 \tilde{5}, 42$ p. C. Kohlenston und 5,11 p. C. Wasserstoff im Mittel aus drei Verbrenungen. Die Substanz war bei $100^{\circ} \mathrm{C}$. getrocknet. Dic Zusammensetzung weicht von der, welche die liuherythrinsiture besitzt, in Wasserstolfe gar nicht ab, im Kohlenstoffe dilferint sie $\mathrm{um} 0,89$ p. C. Ob dieses von einer Unreinigkeit herribut oder nicht, liomute ich nicht nachweisen, da ich keine Wurzel von Morinda cilrifolia und kcin Morindin besass. Anderson giebt an, dass sein Morindin ron einem lileinen Kalkgehalte durch Nlkohol, der mit Salzsäure angesãuert war, befreit worden sei. Es wäre sehr leicht möglich, dass dabei eine Spur Alizarin entstanden wäre, welche Beimengung selır gering zu sein braucht, um den Kohlenstoffgehalt um 0,89 p. C. zu erhühen, da las Alizarin bei $100^{\circ} \mathrm{C}$. getrocknet um mels als $14 \mathrm{p}$. C. Kohienstoff mehr enthät, als die Ruberythrinsiuure.

Die Identität des Morindon mit Alizarin ist ferner im höchsten Grade wahrscheinlich. Ich selze die Eigenschaften, so weit sie von Anders on rom Morindon angegeben wurden, neben die entsprechenden Eigenschaften des Alizarin.

\section{Morindon.}

Krystallisirt beim Sublimiren
in rothen Nadeln.

Unlöslich in Wasser, löslich in Alkohol und Aether, aus diesen $\begin{aligned} & \text { Lúsungen beim Verdunsten bry- } \\ & \text { stallisirend. }\end{aligned}$
Alizarin.

Gleichfalls.

Gleichfalls. 
Morindon.
Aliæarin.

Von Alkalien mit prächtig ) violetter Farbe auflöslich.

Mit Baryt-Wasser kobalt- $\}$ Mit Baryt-Wasser lunkel vioblauer Niederschlag.

Färbt mit Thonerde gebeizte Zeuge tief rosenroth, mit Eisenbeize versehene Zeuge violett und schwarz.

Es entsteht aus dem gelben Morindin durch Einwirkung der Schivefelsüure und trokkene Destillation.

Die Analyse, welche $\Lambda \mathrm{nderson}$ mit dem Morindon anstellte gab Zahlen, die der Formel $\mathrm{C}_{20} \mathrm{II}_{7} \mathrm{O}_{\tau}$, also Alizarin + cinem Aequivalent Wasser hesser entspricht, als der, welche A uders on darauf bereclinete. Berechuet. Gef, v. Anderson.

$$
\begin{aligned}
& 20 \text { Aeq. Kohlenstof } 65,57 \quad 65,81 \\
& 7 \text { "Wasserstoif } 3,83 \quad \text { h,18 } \\
& 7 " \text { Sauerstoff } \frac{30,60}{100,00} \quad \frac{30,01}{100,00}
\end{aligned}
$$

Die Morinda citrifolia scheint also denselben Farbstof wic wie die Rubia tinctorum zu enthalten. Anderson hat diese Achnlichkeit bemerkt, konnte sie abcr damals nicht verfolgen, weil die Kenntnisse von den Farbstoffen des Krappes damals äusserst mangelhaft waren. Ich babe erwähnt, dass Anderson auf das Morindin die Formel $\mathrm{C}_{28} \mathrm{H}_{15} \mathrm{O}_{15}$ berechnet hat. Die Analysen der Ruberythrinsäure entsprechen ebensowohl der Formel $\mathrm{C}_{56} \mathrm{H}_{31} \mathrm{O}_{31}$ als der Formel $\mathrm{C}_{72} \mathrm{H}_{40} \mathrm{O}_{40}$ wie die nebenstehende Berechuung zeigt.

\begin{tabular}{|c|c|c|c|}
\hline $\begin{array}{cc}11 & \text { Aeq. } \\
7 & " \\
7 & " \\
2 & "\end{array}$ & $\begin{array}{l}\text { Bohlenstoff } \\
\text { Wasserstoff } \\
\text { Sauerstoff } \\
\text { Bleioxyd }\end{array}$ & $\begin{array}{c}\text { erechnet. } \\
22,70 \\
1,89 \\
15,13 \\
60,20\end{array}$ & $\begin{array}{c}\text { Gefunden. } \\
22,74 \\
2,00 \\
15,82 \\
5 y, 44\end{array}$ \\
\hline & & $\overline{100,00}$ & $\overline{100,00}$ \\
\hline
\end{tabular}

$$
\begin{aligned}
& 56 \text { Aeq. Kohlenstoff } \begin{array}{c}
\text { Berechnet. Gefunden. } \\
54,64 \\
55,54 \\
54,42
\end{array} \\
& 31 \text { " Wasserstoff } 5,04 \quad 5,16 \\
& 31 \text { " Sauerstoff } \frac{40,32}{100,00} \quad \frac{40,30}{100,00}
\end{aligned}
$$

Die Analyse des ruberythrinsauren Bleioxyds stimmt ebenso nahe mit der Formel $\mathrm{C}_{14} \mathrm{H}_{7} \mathrm{O}_{\gamma}, 2 \mathrm{PbO}$. 
Ein kleiner Ueberschuss an Ruberythrinsäure und dagegen eine kleine Menge Bleioxyd weniger wïren durch eine beim Auswaschen beginnende Zersetzung des Salzes erklärlich.

Ziehen wir den Bleioxydgehalt ab, so stimmt die Zusanmensetzung der an das Oxyd gebundenen Substanz genau mit der Formel $\mathrm{C}_{42} \mathrm{H}_{22} \mathrm{O}_{22}$.

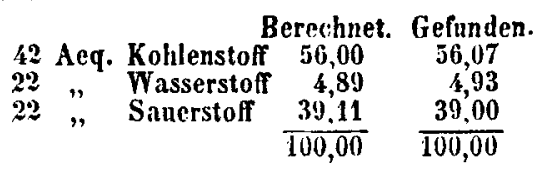

Diese Formel als die richtige angesehen, wäre die rationelle Formel der freien Sãure $\mathrm{C}_{50} \mathrm{H}_{31} \mathrm{O}_{31}=4\left(\mathrm{C}_{14} \mathrm{II}_{7} \mathrm{O}_{7}\right)+3110$. Das Bleisalz wäre $=\mathrm{C}_{\mathbf{1 4}} \mathrm{II}_{7} \mathrm{O}_{7}+2 \mathrm{PJ}, \mathrm{O}$ oder noch genauer wāre die Substanz des Bleisalzes nach der Formel $\mathrm{C}_{42} \mathrm{H}_{22} \mathrm{O}_{22}=3\left(\mathrm{C}_{14} \mathrm{H}_{7} \mathrm{O}_{7}\right)$ + $\mathrm{HO}$ zusammengesetzt. Dic Entstehung von Zucker und Alizarin würde nach der Gleichung vor sich gehen: $\mathrm{C}_{14} \mathrm{II}_{7} \mathrm{O}_{7}=$ $\mathrm{C}_{10} \mathrm{H}_{3} \mathrm{O}_{3}+\mathrm{C}_{4} \mathrm{II}_{4} \mathrm{O}_{4}$. - Die Gruppe $\mathrm{C}_{4} \mathrm{II}_{4} \mathrm{O}_{4}$ würde durch Verdreifachung des Atomgewichtes $\mathrm{C}_{12} \mathrm{II}_{\mathbf{1 2}} \mathrm{O}_{12}=$ Zucker geben, während $\mathrm{C}_{10} \mathrm{II}_{3} \mathrm{O}_{3}$ ein halbes Aequivalent Alizarin reprisentirt.

Dadurch lommt die Ruberythrinsäure in naben Zusammenhang mit den übrigen in der Rubia tinctorum enthaltenen Stoffen. Die Rubichlorsāure, die sich in den Blättern, Stengeln und Wurzeln dieser Pflanze findet, ist nach der Formel $\mathrm{C}_{14} \mathrm{H}_{8} \mathrm{O}_{9}$ zusammengesetzt. Die Gerbsiuuce in den Blăttern hat die $\mathrm{Za}-$ sammenselzung $\mathrm{C}_{14} \mathrm{H}_{8} \mathrm{O}_{9}$. Von dieser Säure findet sich in den Wurzeln eben so wenig eine Spur, als umgekehrt sich in den Blättern keine Spur von Ruberythrinsăure findet. Wāhrend die Gerbsäure der China nova zerfällt in Chinovaroth und Zucker nach dem Schema: $\mathrm{C}_{14} \mathrm{H}_{8} \mathrm{O}_{7}=\mathrm{C}_{2} \mathrm{H}_{2} \mathrm{O}_{2}+\mathrm{C}_{12} \mathrm{H}_{6} \mathrm{O}_{5}$, zerfiele die Ruberytbrinsāure in Zucker und Alizarin in ganz ähnlicher Weise. $\mathrm{C}_{14} \mathrm{H}_{7} \mathrm{O}_{7}=\mathrm{C}_{4} \mathrm{II}_{4} \mathrm{O}_{4}+\mathrm{C}_{10} \mathrm{H}_{3} \mathrm{O}_{3}$. Die Entstehuug der Ruberythrinsäure scheint in den Stengeln der Rubia tinctorum stattzufinden. Während die untersten Theile der Stengel gelbe Farbestoffe enthalten, ist in der oberen Hälfte derselben nichts davon zu bemerken. Werden die Stengel zerquetscht so nehmen sie nach einiger Zeit an der Luft eine rothe Farbe an.

Die Menge der Ruberythrinsāure betrāgt in der Wurzel des Krappes nicht mehr als beilaufig $1 \mathrm{Gramm}$ auf 25 Pfd. der 
luftrockenen Wurzel; der levantinische Krapp enthält mehr als der in Europa gebaute von dieser Säure.

Ich babe in der ersten Abtheilung dieser Arbeit gezeigt, dass alles Alizarin und Purpurin durch neutrales essigsaures Bleioxyd aus einem wässerigen Krappdecoct ausgefällt werde. In ähnlicher Weise lässt sich Barytwasser anwenden. Eiu Krappdecoct giebt mit Barytwasser einen violetten Niederschlag, der, abfiltrirt und mit mãssig starker Salzsäure ũbergossen, eine Lösung giebt, die chlorbaryumhaltig ist, während das Alizarin und etwas Purpurin zurückbleiben, die durch Umkrystallisiren gereinigt werden können.

Ich habe die Einwirkung von Ammoniakgas auf Alizarin bei erhöhter Temperatur beobachtet. In einer Kugelröhre befindliches Alizarin erwärmt sich, wenn Ammonialigas darüber geleitet wird und färbt sich violett. Bein Erhitzen wird das Ammoniak ausgetrieben und das Alizarin nimmt seine gelbe Farhe wieder an. Wird das Alizarin im Strome von Ammoniak bis zur Verlüchtigung erhitzt, so bleibt etwas Kohle und man erhält ein violettes pulverförmiges Sublimat, das in Wasser nur wenig löslich ist. Eine kleine Menge Ammoniak zugesetzt, löst es sich mit der Farbe des Mlizarin auf und durch Zusatz von Säuren werden orangegelbe Flocken von Alizarin gefallt.

In dem Krapp sind alsa folgende Stofre enthalten:

Citronsāure, Pectinsāure, Ruberythrinsāure, Alizarin, Purpurin, Zucker, die letzteren drei Kürper wahrscheinlich durch Zersetzung der Ruberythrinsăure gebildet, und Rubichlorsāure.

\section{XII. \\ Notiz über die Kaffeebohnen. \\ Von \\ Prof. Rochleder.}

(Sitzungsbericht d. Kais. Akad. d. Wissensch. vom 11. Decbr. 1851.)

Schon vor lăngerer Zeit hat Schrader bei der Untersuchung der Kaffeebohnen die Beobachtung gemacht, dass der eigenthümliche Extractivstoff des Kaffees, d. h. ein Gemenge von 